\title{
DARK CLOUD OBSERVATIONS: PROBLEMS AND PROGRESS
}

\author{
Y. C. MINH \\ Korea Astronomy Observatory \\ Hwaam, Yusong, Taejon 305-348, Korea
}

\begin{abstract}
Interstellar dark clouds have provided a laboratory which has been successfully used to identify a number of species, perform high resolution spectroscopy, and characterize the processes in the interstellar medium. We have successfully solved a number of problems and achieved significant progress through dark cloud observations. With the improvement of observing techniques and theories, however, we are now having new problems and are waiting for further progress. The dark clouds, because of their quietness and large column densities, still provide a very useful testing ground of many uncertain interstellar processes. Here we summarize some of the recent progress made in dark cloud observations and discuss related problems. We focus especially on the importance of nearby objects, possible signs of gas-grain interactions in dark clouds, and further sensitive observations.
\end{abstract}

\section{Introduction}

Our picture of the interstellar medium has been improved very rapidly since the first detection of $\mathrm{CO}$ in interstellar clouds. The dark clouds have been used to characterize the molecular probes of various physical parameters and to test those interstellar processes which will consequently determine the evolution of the interstellar clouds. Considerable progress has been made in understanding the interstellar phenomena. The interstellar medium is, however, very complex and consists of objects of very different characteristics, such as diffuse clouds, quiescent dark clouds, star-forming regions, shock regions, photon-dominated regions, etc. The properties of molecular clouds span a large range in size, density and mass with structures on all spatial scales. They are also chemically inhomogeneous (cf. Irvine et al. 1987). With the progress of observing techniques and theories, 
we are having a new look on the interstellar medium. The quiescent dark clouds may still be very useful laboratories to characterize many uncertain interstellar processes.

In this paper we discuss some selected topics related to dark cloud observations. In $\S 2$, we treat observations of nearby objects and discuss their molecular abundances. In $\S 3$ we introduce, as signs of gas-grain interactions, the ortho/para ratios and the abundances of some oxygen- and sulfur-containing species. The need for further sensitive observations is discussed in $\S 4$.

\section{Observations of nearby objects}

The ISM has shown large density fluctuations on various spatial scales, probably to the limit which the telescopes can resolve, and higher and higher angular resolutions have been required for an improved understanding of the ISM. The nearby high-latitude clouds (HLCs) are located at distances of about $100 \mathrm{pc}$ from the sun (Blitz et al. 1984) and also extend to a few square degrees. The 1 arcminute resolution, for example, corresponds to a linear distance of $0.02 \mathrm{pc}$ at the distance of MBM12, the nearest molecular cloud (distance only $\sim 65 \mathrm{pc}$ from the sun). The HLCs are usually distinguished from the denser clouds located in the Galactic plane by their low visual extinctions $\left(A_{v} \sim 1-2 \mathrm{mag}\right)$ and small masses $(10-100$ $\left.\mathrm{M}_{\odot}\right)$. HLCs span a wide range of physical and chemical properties and fall into the category known as translucent clouds (cf. van Dishoeck \& Black 1988). These clouds provide a unique opportunity to study the physical and chemical properties of interstellar clouds in detail.

Although there exist considerable uncertainties in the molecular abundance data, recent observations suggest that the molecular abundances of the HLCs are very similar to typical dense dark clouds and there may be no need for a distinction between diffuse clouds and dense molecular clouds (Minh et al. 1996; Turner 1996, and references therein). Some of the observational distinction could result from different observing conditions. Some discussions on the abundance uniformity and gas-phase reactions and role of grains in quiescent gas clouds have appeared (e.g. Turner 1996). It may not be proper, however, to say that abundance uniformity exists over a large range of cloud densities and temperatures. We may rather say that the uniformity could exist in the basic unit of dense clumps and probably we can define the physical and chemical properties of these clumps. Then the distinctions among interstellar clouds can be interpreted, in part, with filling factors of clumps and interclump medium. The fragmentary structure and turbulence in a clumpy medium have been observed and extensively discussed, for instance, by Falgarone et al. (1992). The filling factor of the 

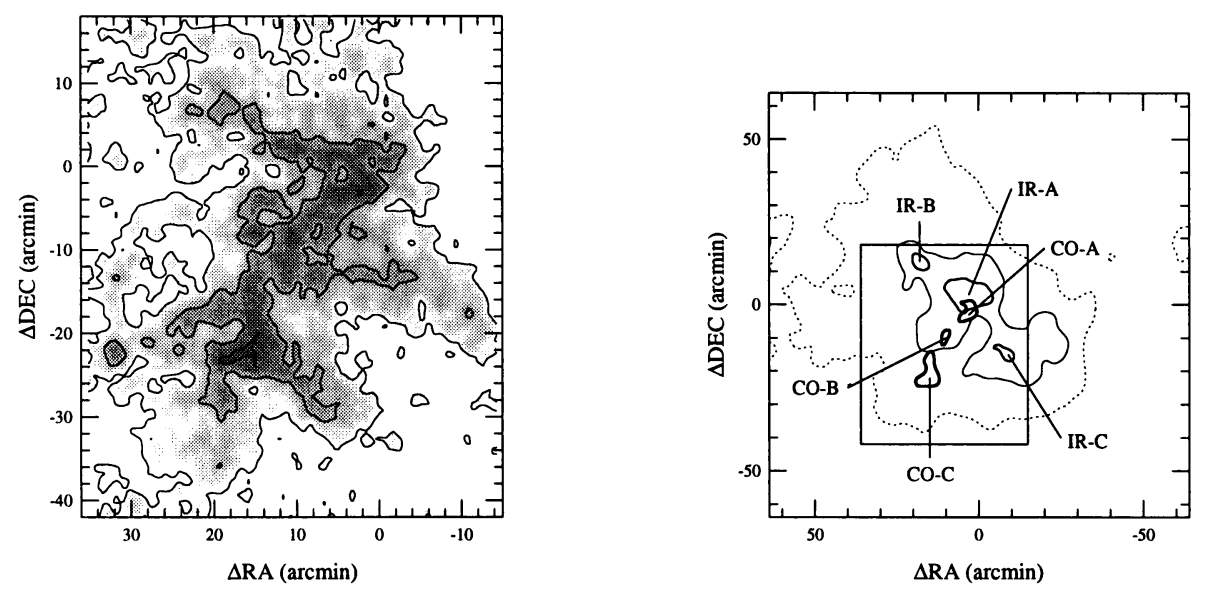

Figure 1. Integrated intensity map (right) of the CO (1-0) line, and the half-power contours of the observed species (right) toward the high latitude cloud MBM7 (Minh et al. 1996).

observed species could be an important parameter which characterizes the cloud and hence should be examined seriously if the observed species are compared with each other, or with model calculations.

One example of a highly fragmented region is B5, which is located at $\sim 350 \mathrm{pc}$ from the sun and has low-mass star forming cores embedded in a less dense interclump medium (Goldsmith et al. 1986). The recycling of material between the dense clumps and the tenuous interclump medium has been modelled and its implications have been discussed extensively (e.g. Charnley et al. 1988). This process is especially important for the chemical evolution of interstellar clouds. Fig. 1 is an another example of a clumpy cloud, MBM7, which is one of the HLCs listed in Magnani et al. (1985). It is located $\sim 150 \mathrm{pc}$ from the sun and extends to $\geq 4$ square degrees in angular size. MBM7 shows a very clumpy structure with a total mass $\sim 30$ $\mathrm{M}_{\odot}$ (Minh et al. 1996). As shown in Figure 1, we can here very easily distinguish dense clumps of sizes less than $0.1 \mathrm{pc}$. Probably we can also identify the atomic to molecular transition zone in this source. Fig. 2 shows observed spectra toward MBM12 and 2-component cloud model calculations using a three dimensional Monte Carlo code (Park et al. 1996). The observed CO line profiles toward MBM12 have been successfully reproduced by this 2-component model. This is just an example of several recent model calculations aiming at understanding the clumpy nature of these molecular clouds (e.g. Tauber et al. 1991). The HLCs must be the ideal source to study the clumpy structure because of their proximity, large angular sizes, and relatively low masses, in addition to the less confusion with foreground 


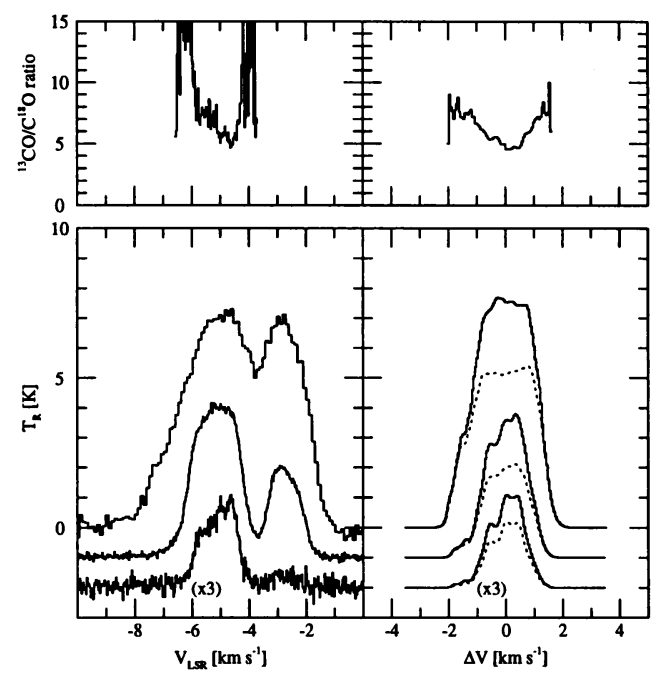

Figure 2. The observed line profiles (left) of 1-0 transitions of ${ }^{12} \mathrm{CO},{ }^{13} \mathrm{CO}$, and $\mathrm{C}^{18} \mathrm{O}$ toward MBM12, and the model calculations (right) (see details in Park et al. 1996).

and background emissions.

\section{Signs of gas-grain interactions?}

The hot cores in star-forming regions are showing clear signs of evaporation of grain mantles, which must have been accreted during their dark cold cloud phase (e.g. Blake et al. 1987). The infrared absorption features of the grain mantle constituents observed toward continuum sources also suggest previous interactions between gas and grains (cf. Tielens \& Whittet, in this volume). Although these are direct evidences of the existence of grain mantles in molecular clouds, we have very few signs that gas-grain interactions are actually taking place in the quiet gas phase. Anyhow it has been suggested that collisions between gas and grains would lead to the removal of molecules from the gas phase in times considerably shorter than the lifetimes of the molecular clouds. Thus, various desorption mechanisms and dynamical models have been proposed to release the molecules (cf. Léger et al. 1985), although their efficiencies are still controversial. We may, however, agree that the grain-related processes can be very active in cold dark clouds, and if we find some evidence of gas-grain interactions in dark clouds, we can constrain the grain-related processes much better. One of the indirect evidences may be the observed ortho/para ratio in dark clouds. The abundances of some oxygen- or sulfur-containing species may also indicate the importance of grain-processes. 


\subsection{ORTHO-TO-PARA RATIOS}

A molecule with two identical nuclei can have two distinct states, ortho and para, depending on the spin states of these nuclei. The abundance ratio between these two states has been thought to provide information on molecular formation processes, since radiative or collisional conversion from one form to the other is forbidden. The molecular formation temperatures, in general, are much larger than the difference between the ortho and para rotational ground states. Therefore, when molecules form, the $o / p$ abundance ratio would be given by the ratio of statistical weights of the two species (the high temperature equilibrium value). If the measured $o / p$ ratio of a species deviates from the high temperature equilibrium value, we may think that other processes must have been effective subsequent to the formation of the species, such as proton exchange reactions in the gas phase or on the grain surfaces.

Molecular hydrogen has been studied in high-temperature regions to determine its ortho/para ratio, but $\mathrm{H}_{2}$ is hard to observe in dense molecular clouds with kinetic temperatures of $10-100 \mathrm{~K}$ since it has no permanent electric dipole moment. In dense molecular clouds the $\mathrm{o} / \mathrm{p}$ ratios have been studied for $\mathrm{H}_{2} \mathrm{CO}, \mathrm{H}_{2} \mathrm{CS}, \mathrm{H}_{2} \mathrm{CCO}, \mathrm{C}_{3} \mathrm{H}_{2}, \mathrm{H}_{2} \mathrm{C}_{3}, \mathrm{H}_{2} \mathrm{C}_{4}$, etc (cf. Minh et al. 1995). $\mathrm{CH}_{3} \mathrm{CN}$ is an another example having ortho $(E)$ and para $(A)$ states and its observed $\mathrm{o} / \mathrm{p}$ ratio indicates a value thermalized to kinetic temperature (Minh et al. 1993). Fig. 3 shows the observed spectrum of the $\mathrm{CH}_{3} \mathrm{CN} J=2-1$ lines. Since the ortho and para lines appear in the same antenna beam and frequency band, we can cancel a lot of observational uncertainties by comparison of the line intensities, and in addition, the different $K$-component lines enable a better estimate of the excitation conditions. We always need precise abundance measurements to compare the ortho and para abundances, which only became available with improved sensitivity of the receivers. The modelling of the $\mathrm{CH}_{3} \mathrm{CN}$ o/p conversion on the surface of interstellar grains seems to explain the observed ratio very well (Willacy et al. 1993).

This ortho/para ratio has limitations, such as requirements for more precise abundance measurements, better understanding of gas phase proton exchange reactions (which are largely uncertain), and proper desorption processes to claim the interaction with grains. However, since we have very few direct observational results on the gas-grain interactions in quiet gas clouds, the $o / p$ ratio could give useful constraints on grain processes with further studies. It will also be interesting to see the dependence of the $\mathrm{o} / \mathrm{p}$ ratio on the physical conditions of the sources. And even in hot cores the $\mathrm{o} / \mathrm{p}$ ratio may give some constraints on the grain processes of the previous dark cloud phase. 


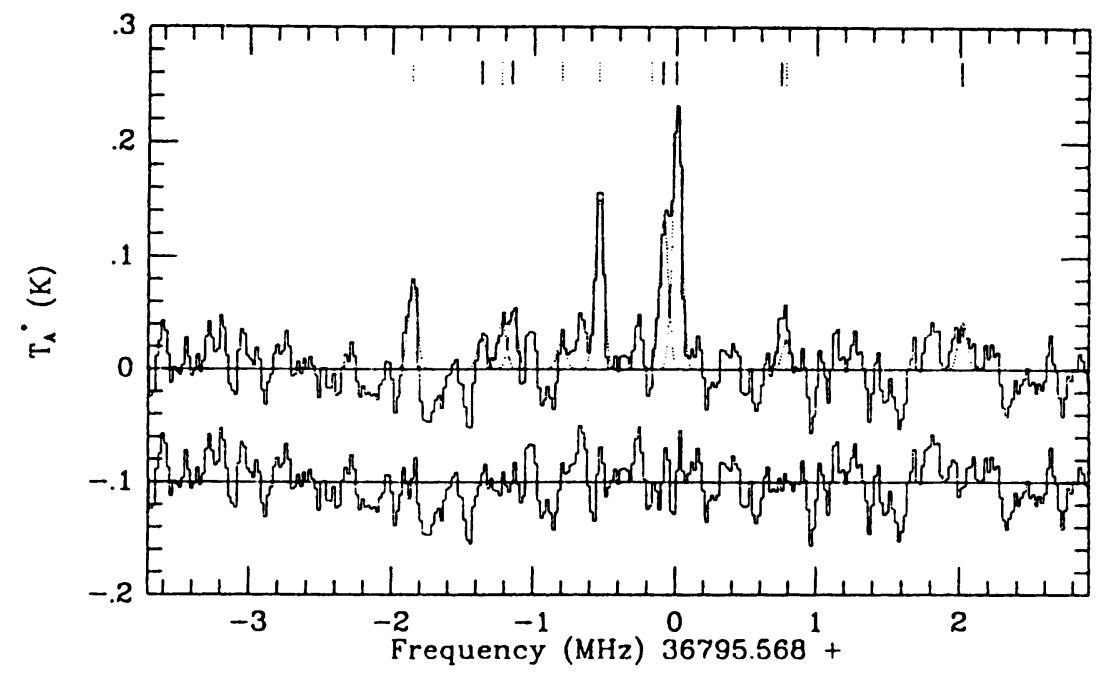

Figure 3. The $J=2-1$ methyl cyanide spectrum obtained toward TMC-1. The bars at the top indicate the rest frequencies for the $E$ (dotted) and $A$ (solid) transitions. Gaussian fit results for the spectrum are shown as dotted lines, with the residuals presented below the observed spectrum (Minh et al. 1993).

\subsection{OXYGEN AND SULFUR CHEMISTRY}

Oxygen is the most abundant element after helium in the ISM but the repository of oxygen is still an open question in interstellar chemistry. Poglitsch et al. (1996) have found a large abundance of atomic oxygen $\left([\mathrm{O}] /[\mathrm{H}] \geq 6 \times 10^{-4}\right)$ in a dark cloud observed as an absorption of the 63 $\mu \mathrm{m}$ fine-structure line of atomic oxygen toward DR21. This result is in sharp contrast to predictions of steady state chemistry models, and suggests that a large fraction of the gas-phase oxygen may be in atomic form. This result also emphasizes the importance of high spectral resolution observations toward dark clouds. Another important constraint of oxygen chemistry would be given by the abundances of $\mathrm{H}_{2} \mathrm{O}$ and $\mathrm{O}_{2}$, both of which have not been detected in the quiescent gas phase. The space telescopes, which are already working in space or planned to be launched soon, such as ISO, ODIN, and SWAS, could give, at least, good limits on the abundances of water vapor and molecular oxygen in dark clouds. If we can compare the gas-phase water abundances with the onset of the ice-band appearance in dark clouds from the satellite observations, it will give a very important constraint on the oxygen chemistry and gas-grain interactions. 

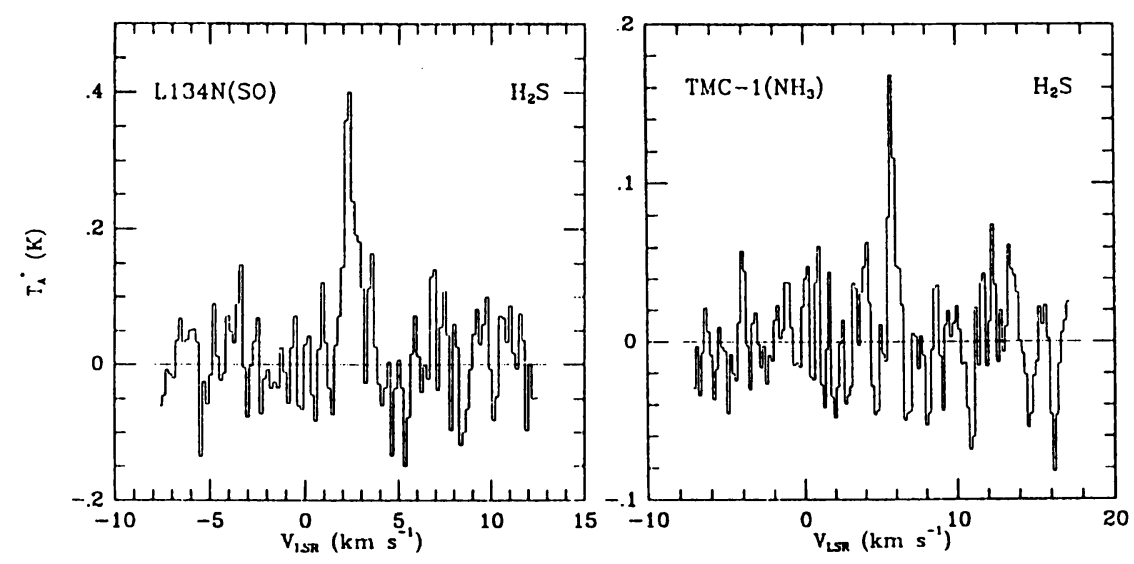

Figure 4. Spectra of the $1_{10}-1_{01}$ transition of $\mathrm{H}_{2} \mathrm{~S}$ observed toward the dark clouds L134N (left) and TMC-1 (right) (Minh et al. 1989).

Another long standing problem is the low abundance of sulfur species in quiescent gas clouds, which results from a large depletion of sulfur in the gas phase and the endothermicity of the formation reactions of several major sulfur-containing species (cf. Watson \& Walmsley 1982; Prasad \& Huntress 1982; Millar \& Herbst 1990). Therefore, the abundances of some sulfur-containing molecules have been thought to result from either grainsurface reactions or shock reactions. Fig. 4 provides an example of a sulfur molecule detected in cold dark clouds. The existence of $\mathrm{H}_{2} \mathrm{~S}$ in these clouds may indicate formation of this species on grain surfaces (Minh et al. 1989). Gas-phase sulfur chemistry is, however, not well known, and there is a controversy that ion-molecule chemistry can explain the abundances of the sulfur-containing species, at least in translucent clouds (Turner 1996). We still need much improved observations and theoretical considerations on the sulfur-related reactions. Especially the observations of radicals, such as $\mathrm{OH}$, $\mathrm{CH}$, and $\mathrm{SH}$, will be important in understanding the gas phase reactions together with $\mathrm{H}_{2} \mathrm{O}$ and $\mathrm{H}_{2} \mathrm{~S}$ observations.

\section{Toward more sensitive observations}

We have discussed observations of nearby objects in $\S 2$. These nearby objects are often extended to a few square degrees with relatively weak molecular emissions. Focal plane arrays with very sensitive receivers, such as QUARRY of FCRAO, are powerful tools in imaging these nearby objects. High spectral resolution spectrometers are also required. We are expect- 
ing more sensitive millimeter-, centimeter-wave and infrared observations in studying these objects.

One of the other subjects which needs more sensitivity is the clear detection of collapsing (or expanding) features of dense cores. About $50 \%$ of the dense $\mathrm{NH}_{3}$ cores are associated with IRAS sources, but there are very few observations which show evidence for collapsing cores in dark clouds (cf. Myers \& Benson 1983; Zhou et al. 1993; Velusamy et al. 1995). These observations need high spatial resolutions ( $\sim$ a few arcseconds) with more sensitivity than reported at present. In addition, we have to choose proper tracers to detect collapsing profiles. Zhou (in this volume) gives a specific discussion on the collapsing cores and Shu (in this volume) also suggests some tracers. We suggest that $\mathrm{HC}_{3} \mathrm{~N}$ could be a good tracer of the collapsing cores since this species is thought not to be distributed widely but resides mostly in very dense cores, and its abundance may be enhanced by the activities of the embedded sources. These observations will give constraints on the collapse time scale and also on the active role of grain chemistry, which are important in physical and chemical models of the cloud evolution.

Finally, we briefly mention the absorption line measurements toward dark clouds. While molecular emission lines come preferentially from dense clumps, the absorption against the continuum source would occur mostly by extended diffuse gas clouds. The absorption line measurements can give us a definite clue in understanding the structure and composition of the interclump medium. Unlike the infrared and higher frequency regions, we have very few data at radio wavelengths. The reader is referred to Lucas \& Liszt (in this volume) for some aspects of recent results. There are now very sensitive millimeter-wave interferometers, as well as centimeter-wave arrays, together with a large number of strong background continuum sources. Probably we are opening a new important window to understand the nature of dark clouds.

\section{Concluding remarks}

Although we now have a much better picture of interstellar conditions compared to 10 years ago or even 5 years ago, interstellar chemistry appears to be becoming more complex with the progress of observing techniques and theories. The cold dark clouds, which provide the connection from the diffuse gas to the active star-forming regions, still provide the best laboratories, because of their quietness and large column densities, to study many of the uncertain interstellar processes. Substantial observational improvements can still be made toward dark clouds and, of course, together with good theories to explain the results, we can certainly give much better constraints on the interstellar processes. 


\section{Acknowledgements}

This manuscript was written mostly while the author was taking a sabbatical leave at the Onsala Space Observatory at Sweden. It is a pleasure to thank the staff of the OSO for their hospitality, and Åke Hjalmarson for his critical reading of the manuscript.

\section{References}

Blake, G. A., Sutton, E. C., Masson, C. R., Phillips, T. G. 1987, ApJ 315, 621

Blitz, L., Magnani, L., Mundy, L. 1984, ApJ 282, L9

Charnley, S. B., Dyson, J. E., Hartquist, T. W., Williams, D. A. 1988, MNRAS 231, 269

Falgarone, E., Puget, J. L., Perault, M. 1992, A\&A 257, 715

Goldsmith, P. F., Langer, W. D., Wilson, R. W. 1986, ApJ 303, L11

Irvine, W. M., Goldsmith, P. F., Hjalmarson, $\AA$. 1987, in Interstellar Processes, eds. D. J. Hollenbach \& H. A. Thronson Jr. (Dordrecht: Reidel), p. 561

Léger, A., Jura, M., Omont, A. 1985, A\&A 144, 147

Magnani, L., Blitz, L., Mundy, L. 1985, ApJ 295, 402

Millar, T. J., Herbst, E. 1990, A\&A 231, 466

Minh, Y. C., Dickens, J. E., Irvine, W. M., McGonagle, D. 1995, A\&A 298, 213

Minh, Y. C., Irvine, W. M., Ohishi, M., Ishikawa, S., Saito, S., Kaifu, N. 1993, A\&A 267, 229

Minh, Y. C., Irvine, W. M., Ziurys, L. M. 1989, ApJ 345, L63

Minh, Y. C., Park, Y.-S., Kim, K.-T., Irvine, W. M., Brewer, M. K., Turner, B. E. 1996, ApJ 467, 717

Myers, P. C., Benson, P. J. 1983, ApJ 266, 309

Park, Y.-S., Hong, S. S., Minh, Y. C. 1996, A\&A 312, 981

Poglitsch, A., Herrmann, F., Genzel, R., Madden, S. C., Nikola, T., Timmermann, R., Geis, N., Stacey, G. J. 1996, ApJ 462, L43

Prasad, S. S., Huntress, W. T. 1982, ApJ 260, 590

Tauber, J. A., Goldsmith, P. F., Dickman, R. L. 1991, ApJ 375, 635

Turner, B. E. 1996, ApJ 461, 246

van Dishoeck, E. F., Black, J. H. 1988, ApJ 334, 771

Velusamy, T., Kuiper, T. B. H., Langer, W. D. 1995, ApJ 451, L75

Watson, W. D., Walmsley, C. M. 1982, in Regions of Recent Star Formation, eds. R. S. Roger \& P. E. Dewdney (Dordrecht: Reidel), p.397

Willacy, K., Williams, D. A., Minh, Y. C. 1993, MNRAS 263, L40

Zhou, S., Evans N. J., Kompe, C., Walmsley, C. M. 1993, ApJ 404, 232 


\section{Discussion}

Irvine: What are the physical conditions, density and temperature, in clouds like MBM7?

Minh: Densities are about $2000 \mathrm{~cm}^{-3}$ in dense cores, and about an order of magnitude less in the interclump medium. Temperatures are about $10 \mathrm{~K}$ in the core and about $30 \mathrm{~K}$ in interclump media.

Herbst: The $\mathrm{o} / \mathrm{p}$ abundance ratios are determined by the details of reactions. Example:

$$
\begin{gathered}
\mathrm{H}^{+}+\mathrm{H}_{2} \mathrm{CO} \rightarrow \mathrm{H}_{3} \mathrm{CO}^{+} \rightarrow \mathrm{H}^{+}+\mathrm{H}_{2} \mathrm{CO} \\
\mathrm{H}^{+}+\mathrm{H}_{2} \mathrm{CO} \rightarrow \mathrm{H}_{2} \mathrm{COH}^{+} \rightarrow \mathrm{H}^{+}+\mathrm{H}_{2} \mathrm{CO}
\end{gathered}
$$

Only (1) allows o/p conversion.

Minh: Thanks for the comment. Yes, I agree that we have to look very carefully at gas-phase proton exchange reactions.

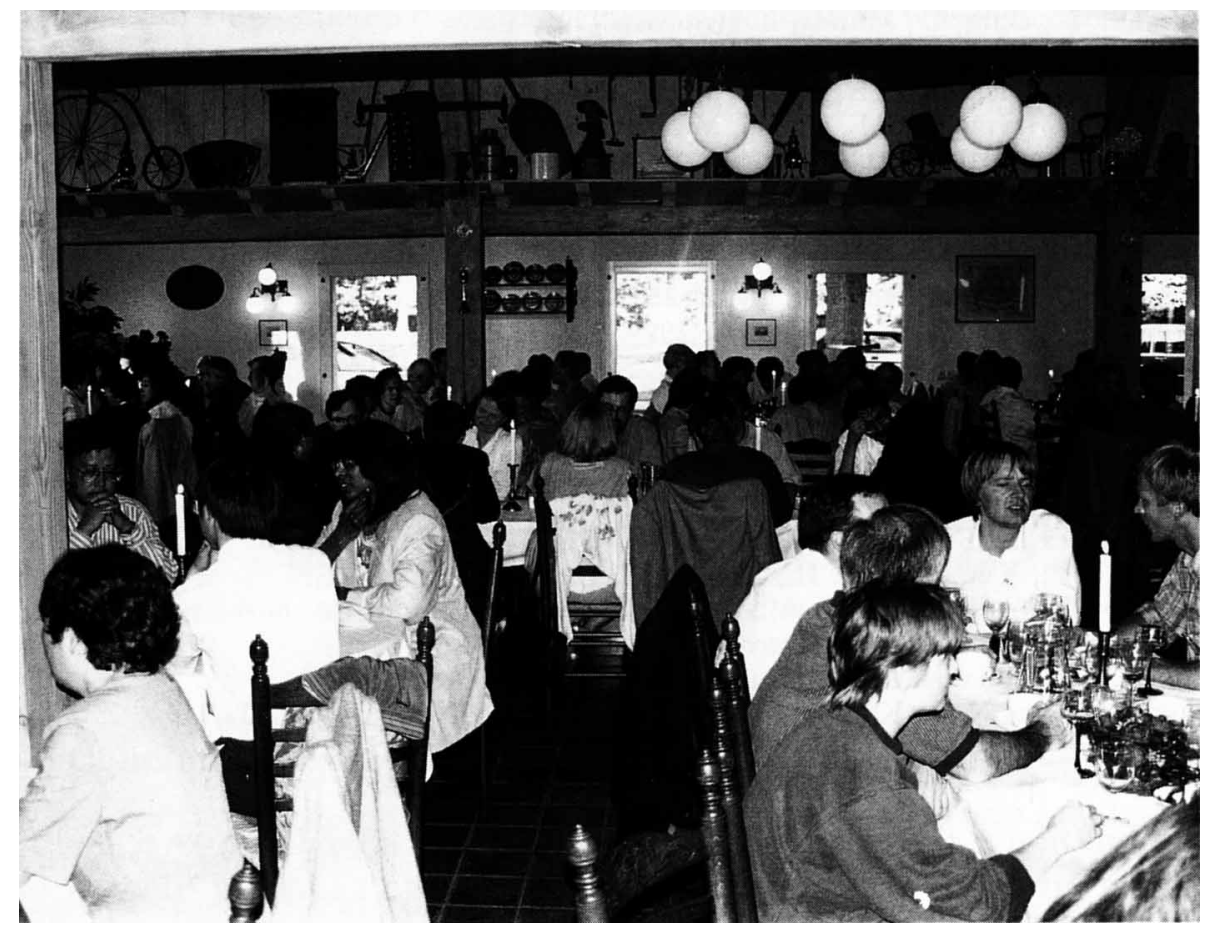

